\title{
Protective effect of Ocimum basilicum essential oil against acetic acid-induced colitis in rats
}

\author{
Vahid Nikoui ${ }^{1}$, Amir Rashidian ${ }^{2}$, Saeed Mehrzadi ${ }^{1}$, Parnia Roohi ${ }^{3}$, Ali Reza Ghannadi ${ }^{4}$ \\ Mohsen Minaiyan ${ }^{3}$, Muhammad Imran Khan ${ }^{5}$
}

${ }^{1}$ Razi Drug Research Center, Iran University of Medical Sciences, Tehran, Iran, ${ }^{2}$ Department of Pharmacology, School of Medicine, Tehran University of Medical Sciences, Tehran, Iran, ${ }^{3}$ Department of Pharmacology, School of Pharmacy and Pharmaceutical Sciences, Isfahan University of Medical Sciences, Isfahan, Iran, ${ }^{4}$ Department of Pharmacognosy, School of Pharmacy and Pharmaceutical Sciences, Isfahan University of Medical Sciences, Isfahan, Iran, ${ }^{5}$ Department of Pharmacy, Kohat University of Science and Technology, 26000 Kohat, KPK, Pakistan

Background: Ocimum basilicum L. has been traditionally used for treatment of inflammatory bowel disease in Iran. This study investigates the ameliorative effect of Ocimum basilicum essential oil on an acetic acid-induced colitis model in rats. Methods: Male rats divided into several groups. The sham group received normal saline $(1 \mathrm{ml})$ without induction of colitis; control group received vehicle ( $1 \mathrm{ml}, 0.2 \%$ Tween 80$)$ two hours before induction of colitis; dexamethasone group received dexamethasone $(2 \mathrm{mg} / \mathrm{kg})$ two hours prior to induction of colitis. The test groups received Ocimum basilicum essential oil at doses of 100, 200, and $400 \mathrm{ml} / \mathrm{kg}$ two hours before induction of colitis. All treatments were performed for the following 5 days, orally. The colon was assayed macroscopically and histopathologically, and myeloperoxidase activity was measured in colon. The essential oil of Ocimum basilicum was also analyzed by gas chromatography-mass spectrometry (GC/MS). Group differences were calculated by parametric one-way analysis of variance with Tukey's posthoc test. Nonparametric data were analyzed using Kruskal-Wallis followed by Mann-Whitney U test. For all tests, differences with values of $\mathrm{P}<0.05$ were considered significant.

Results: Ocimum basilicum essential oil at two doses (200 and $400 \mathrm{ml} / \mathrm{kg}$ ) significantly ameliorated wet weight/length ratio of colonic tissue compared to control group $(\mathrm{P}<0.05)$. Higher doses of essential oil $(200 \mathrm{and} 400 \mathrm{ml} / \mathrm{kg})$ significantly reduced ulcer severity, ulcer area, and ulcer index. On the other hand, histological examination revealed the diminution of total colitis index as a marker for inflammatory cell infiltration in the colonic segments of rats treated with Ocimum basilicum essential oil (200 and $400 \mathrm{ml} / \mathrm{kg}$ ). The increased level of myeloperoxidase was significantly decreased after treatment with the essential oil of Ocimum basilicum (200 and $400 \mathrm{ml} / \mathrm{kg}$ ).

Conclusions: These results suggest that Ocimum basilicum exerts protective effect against acetic acid-induced colitis in rats. 